\title{
Endoscope-assisted supracerebellar transtentorial approach to the posterior medial temporal lobe for resection of cavernous malformation
}

\author{
M. Yashar S. Kalani, MD, PhD, Ting Lei, MD, Nikolay L. Martirosyan, MD, Mark E. Oppenlander, \\ MD, Robert F. Spetzler, MD, and Peter Nakaji, MD
}

Department of Neurosurgery, Barrow Neurological Institute and St. Joseph's Hospital and Medical Center, Phoenix, Arizona

The mesial temporal lobe can be approached via a pterional or orbitozygomatic craniotomy, the subtemporal approach, or transcortically. Alternatively, the entire mesial temporal lobe can be accessed using a lateral supracerebellar transtentorial (SCTT) approach. Here we describe the technical nuances of patient positioning, craniotomy, supracerebellar dissection, and tentorial disconnection to traverse the tentorial incisura to arrive at the posterior mesial temporal lobe for a cavernous malformation. The SCTT approach is especially useful for lesions in the dominant temporal lobe where an anterolateral approach may endanger language centers or the vein of Labbé.

The video can be found here: https://youtu.be/D8mIR5yeiVw.

KEYWORDS cavernous malformation; lateral supracerebellar transtentorial approach; mesial temporal lobe; video 